\title{
Stepwise Inverse Consistent Euler's Scheme for Diffeomorphic Image Registration
}

\author{
Akshay Pai ${ }^{1}$, Stefan Sommer ${ }^{1}$, Sune Darkner ${ }^{1}$, Lauge Sørensen ${ }^{1}$, Jon Sporring ${ }^{1}$ \\ and Mads Nielsen ${ }^{1,2}$ \\ 1 DIKU, University of Copenhagen, Copenhagen \\ 2 Biomediq A/S, Copenhagen
}

\begin{abstract}
Theoretically, inverse consistency in an image registration problem can be achieved by employing a diffeomorphic scheme that uses transformations parametrized by stationary velocity fields (SVF). The displacement from a given SVF, formulated as a series of self compositions of a transformation function, can be obtained by Euler integration in the time domain. However in practice, the discrete time integration produces results that are inverse inconsistent, and inverse consistency in the final solution needs to be explicitly ensured. One way of achieving this is to penalize the endpoint displacement offset obtained by evaluating a composition of the transformation with its inverse at an arbitrary point. In this paper, we propose a variation in which the displacement penalization is required only in the first composition step of the transformation thereby bringing down the computational complexity. We compare these two ways of enforcing inverse consistency by applying the registration framework on four pairs of brain magnetic resonance images. We observe that the proposed stepwise scheme maintains both precision and level of inverse consistency similar to the endpoint scheme.
\end{abstract}

\section{Introduction}

Inverse consistency is particularly important in studies where voxel-wise statistics are used to characterize anatomical changes over time [1]. Diffeomorphic (differentiable transformation with differentiable inverse) methods in image registration are attractive because they yield transformations that are invertible. However, inverse consistency is in practice achievable only if the discrete integral of the similarity measure and regularization are symmetrically approximated [2]. Several diffeomorphic approaches have been proposed and the two most prominent among them are: large deformation diffeomorphic metric mapping (LDDMM) [3,4] and Log-Euclidean framework based on stationary velocity fields (SVFs) [6].

In SVF based image registration, paths of diffeomorphism are generated using one parameter subgroups parameterized by SVFs through the Lie group exponential. The Lie group exponential is realized through a series of self compositions of a transformation function [7]. The generated diffeomorphism paths 
are geodesic with respect to the canonical Cartan connection [8]. Applications of SVF have found widespread success in image registration [8-10].

Deformations generated by SVFs are invertible. However, due to discretization errors, inverse consistency needs to be explicitly enforced, most often through a regularization. Inverse consistency has been addressed by [11] where both the forward and backward transformations were jointly estimated by minimizing the displacement offset obtained by composing the forward and backward transformations at an arbitrary point. Other methods that enforce inverse consistency (both diffeomorphic and non-diffeomorphic schemes) are, but not limited to, constraining the transformation [12], penalizing the Jacobian [13], symmetrizing every gradient descent step [14], log-average of forward and backward transformations during optimization [15] and finding a mid-space to make sure the transformations are evenly applied [16] .

In this paper, we propose to use a modified version of the inverse consistency term defined in [11] where we will apply the displacement offset penalization only on the first composition of the flow field (or stepwise scheme) as opposed to using the entire flow field (or endpoint scheme) . Section 2 will briefly introduce the concept of SVFs based image registration, followed by an introduction to the inverse consistency enforcing regularization. In Section 3, we will present a comparison of the proposed stepwise regularization and endpoint regularization by applying the framework on four pairs of brain magnetic resonance images (MRIs).

\section{Registration}

Given an image pair $I_{1}, I_{2}$, registration is formulated as a variational optimization problem, where the cost function that needs to be minimized is represented as,

$$
E\left(I_{1}, I_{2} ; \varphi\right)=\int_{\Omega} M\left(I_{1}\left(\varphi^{-1}\right), I_{2}\right)+\lambda R(\varphi) d \boldsymbol{x}
$$

where $E$ is the overall energy, $M$ is the similarity measure, normalized mutual information (NMI) [17] in this study, $R$ is a regularization term, $\varphi$ is a warp and $\boldsymbol{x}=(x, y, z)$ is a voxel position. Here, we focus on SVF based registration using B-splines where the warp $\varphi$ is parametrized as

$$
\begin{gathered}
\varphi(\boldsymbol{x})=\phi^{1} \text { where }\left\{\begin{array}{l}
\frac{d \phi^{t}}{d t}=B(\boldsymbol{x} ; p) \\
\phi^{0}=\boldsymbol{x}
\end{array}\right. \\
B(\boldsymbol{x} ; p)=\sum_{i=0}^{3} \sum_{j=0}^{3} \sum_{k=0}^{3} \beta_{i}(x) \beta_{j}(y) \beta_{k}(z) p_{i, j, k},
\end{gathered}
$$

where $p$ is the $\mathrm{B}$-spline parameter and $\beta$ is a cubic $\mathrm{B}$-spline basis function. A displacement can be realized as an Euler integration with unit time step [7]. For 
example, given $\mathrm{n}$ steps, the Euler integration approximation will be;

$$
\begin{aligned}
\phi^{\frac{1}{n}} & =\boldsymbol{x}+\frac{B(\boldsymbol{x} ; p)}{n} \\
\phi^{t+\frac{1}{n}} & =\phi^{t} \circ \phi^{\frac{1}{n}} .
\end{aligned}
$$

\subsection{Inverse consistency}

In [11], inverse consistency was enforced by penalizing the displacement error generated after composing a transformation with its inverse. However, in this method, the computation of inverse is a computationally expensive approximation [14]. We instead combine both the forward and backward registration in the same cost function and explicitly compute the inverse transformation, thus removing the lag due to computing the forward and backward transformations sequentially. With the Euler's scheme, inverse consistency can be achieved in two different ways. The first being the endpoint scheme, where the displacement offset is generated by using the entire flow field,

$$
\begin{aligned}
& E_{\text {endpoint }}\left(I_{1}, I_{2} ; \varphi\right)= \\
& \int_{\Omega_{b}} M\left(I_{1}\left(\phi_{b}^{1}\right), I_{2}\right)+\lambda\left\|\boldsymbol{x}-\phi_{f}^{1}\left(\phi_{b}^{1}\right)\right\|^{2} d \boldsymbol{x}+\int_{\Omega_{f}} M\left(I_{1}, I_{2}\left(\phi_{f}^{1}\right)\right)+\lambda\left\|\boldsymbol{x}-\phi_{b}^{1}\left(\phi_{f}^{1}\right)\right\|^{2} d \boldsymbol{x}
\end{aligned}
$$

where $\Omega_{f, b}$ are the region of interest in the source and target images and $\boldsymbol{x} \in$ $\Omega_{f, b}$ depending on the direction of registration. Note that $\varphi=\left\{\phi_{f}, \phi_{b}\right\}$. The advantage of using the Euler's scheme is that, parametrization of the flow field is required only at the first composition. Hence, if the first composition is made inverse consistent, so will their compositions be. Therefore, the cost function (from (5)) for the stepwise scheme can be re-written as,

$$
\begin{aligned}
& E_{\text {stepwise }}\left(I_{1}, I_{2} ; \varphi\right)= \\
& \qquad \int_{\Omega_{b}} M\left(I_{1}\left(\phi_{b}^{1}\right), I_{2}\right)+n^{2} \lambda\left\|\boldsymbol{x}-\phi_{f}^{\frac{1}{n}}\left(\phi_{b}^{\frac{1}{n}}\right)\right\|^{2} d \boldsymbol{x}+ \\
& \int_{\Omega_{f}} M\left(I_{1}, I_{2}\left(\phi_{f}^{1}\right)\right)+n^{2} \lambda\left\|\boldsymbol{x}-\phi_{b}^{\frac{1}{n}}\left(\phi_{f}^{\frac{1}{n}}\right)\right\|^{2} d \boldsymbol{x}
\end{aligned}
$$

where $n$ is the number of compositions used to realize a deformation.

\subsection{Scaling and Squaring}

The scaling and squaring method [6] speeds up the integration of the SVF. Although B-spline based SVF implementations that use scaling and squaring exist [13], scaling and squaring in this context is limited by the fact that a new 
B-spline must be fit at each squaring step. As this fitting cannot be exact, the control over the parametrization is lost and folds may occur. Furthermore, if displacement offsets are to be used for ensuring inverse consistency, the entire velocity field needs to be traversed in both directions to measure the offset. Therefore, we focus on inverse consistency only with Euler integration without scaling and squaring.

\subsection{Volume change computation}

Since the transformations are a composition of B-splines and using Jacobian integration might amplify numerical noise in the deformation, we use cube propagation [18] instead to compute local volume changes.

\section{Experiments}

Four pairs of 1.5T MRIs randomly chosen from the Alzheimer's disease neuroimaging initiative database ( 2 normal controls and 2 mild cognitively impaired) were co-registered. Bias correction and segmentations were done using the Freesurfer crossectional pipeline. The dimension of the images (both bias corrected and segmentations) was $256^{3} \mathrm{~mm}$ with $1^{3} \mathrm{~mm}$ isotropic voxels.

To perform a simple assessment of the methods, only one resolution of control points with a spacing of $\approx 5 \mathrm{~mm}$ was used. The images were filtered with a Gaussian kernel of size $0.2 \mathrm{~mm}$. Number of compositions used were $n=$ $1,2,4,8,12,16$ and 24 . Registrations were run in 3 variations;

- No IC: Using (5) by setting $\lambda=0$,

- Endpoint: Using (5) by setting $\lambda=0.03$,

- Stepwise: Using (6) by setting $\lambda=0.03$,

\subsection{Evaluation metrics}

To evaluate the performance of the registration itself, we will inspect the correlation coefficient between the source and the registered target image [19]. Inverse consistency was checked by computing; the displacement offset $(\Delta x)$ and atrophy error $(\mathrm{AE})$;

$$
\Delta \boldsymbol{x}=\frac{1}{N} \sum_{\Omega}|| \boldsymbol{x}-\phi_{b}^{1}\left(\phi_{f}^{1}(\boldsymbol{x})\right) \|, \mathrm{AE}=\frac{1}{N}\left|\sum_{\Omega}\left(C_{f}-\frac{1}{C_{b}\left(\phi_{f}^{1}(\boldsymbol{x})\right)}\right)\right|
$$

where $C_{f}$ is the forward voxel-wise volume change map computed using cube propgation, $C_{b}\left(\phi_{f}^{1}(\boldsymbol{x})\right)$ is the backward change map transformed to the target domain using the forward transformation, $x$ are random $N\left(25^{3}\right)$ points in the image and $\Omega$ is a region of interest, whole brain in this case. 


\section{Results}

Figure 1 illustrates the mean correlation coefficient between the source and the registered target image over the 4 image registrations. The correlation coefficient improves with the number of compositions for the non-inverse consistent registration scheme. However, the correlation coefficient tends to remain the same with both the inverse consistent schemes, but better than the non-inverse consistent scheme regardless of the number of compositions used.

Initially, we compute displacement offset using the deformations from the inverse inconsistent registration scheme by shooting a set of random points using both the entire flow field and only the first composition of the flow field with the offset multiplied by $n^{2}$. We observed that the latter approximates the former well. AE and the displacement offsets can be seen in Figures 2, 3. Both AEs and displacement offsets are quite similar for both stepwise and endpoint schemes.

The runtimes for both stepwise and non-inverse consistent scheme were the same and lower than the endpoint scheme (a scale up factor of $\approx 3$ ), i.e., for an optimization iteration of a registration (with 8 compositions) run with stepwise/no inverse consistency scheme, the runtime was 10 secs when compared to the 30 secs with the endpoint scheme (single core implementation on a $2.5 \mathrm{Ghz}$ Xeon). It is important to note that the mean numbers presented (correlation coefficient, AE and $\Delta x$ ) were only from the forward runs since the errors were similar with the backward runs.

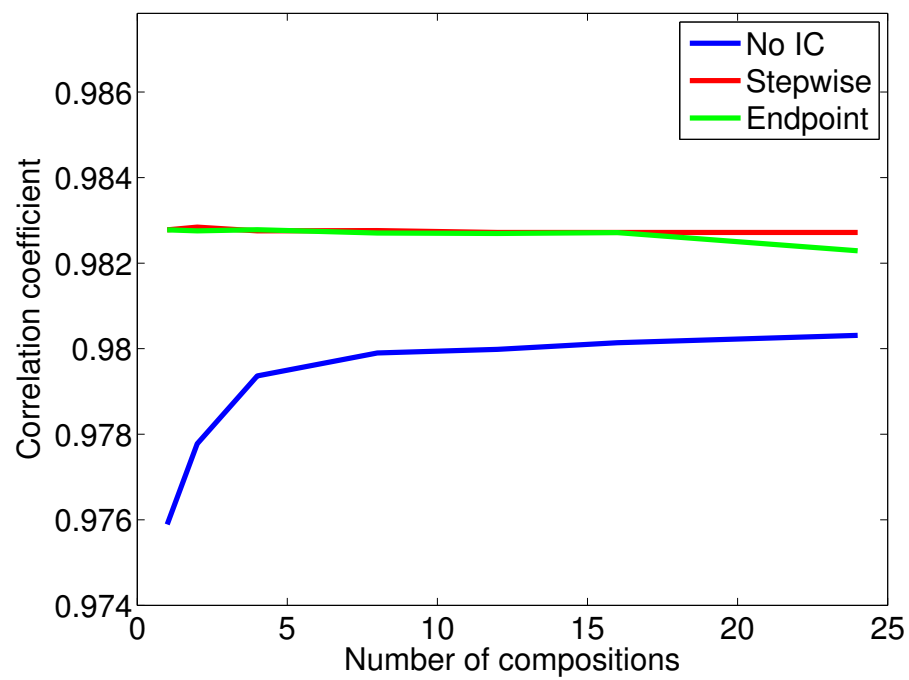

Fig. 1. Mean correlation coefficient between source and registered target image as a function of number of composition 


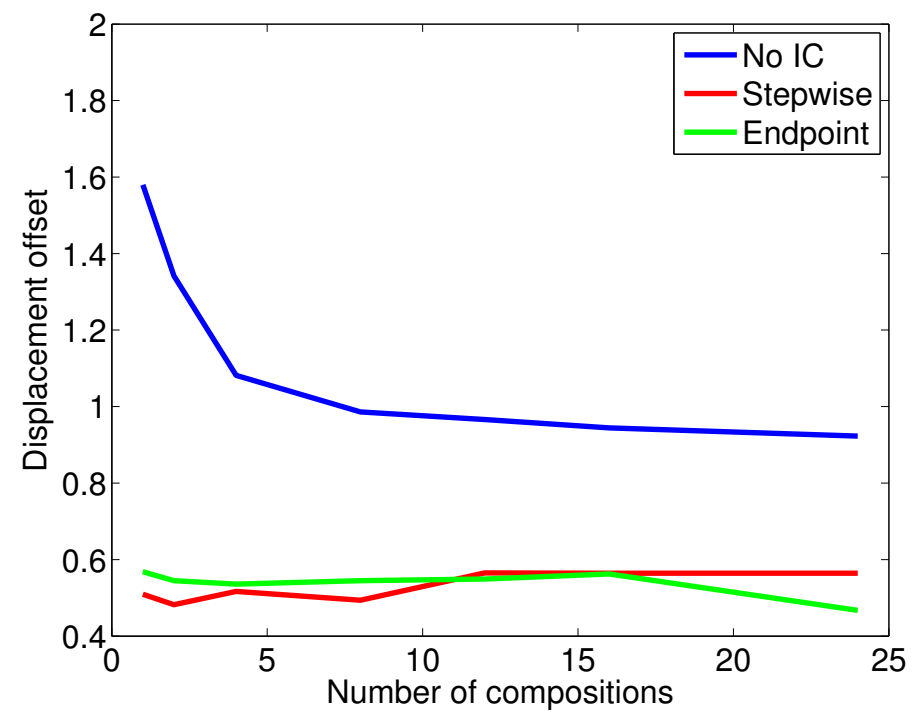

Fig. 2. $\Delta x$ as a function of number of composition

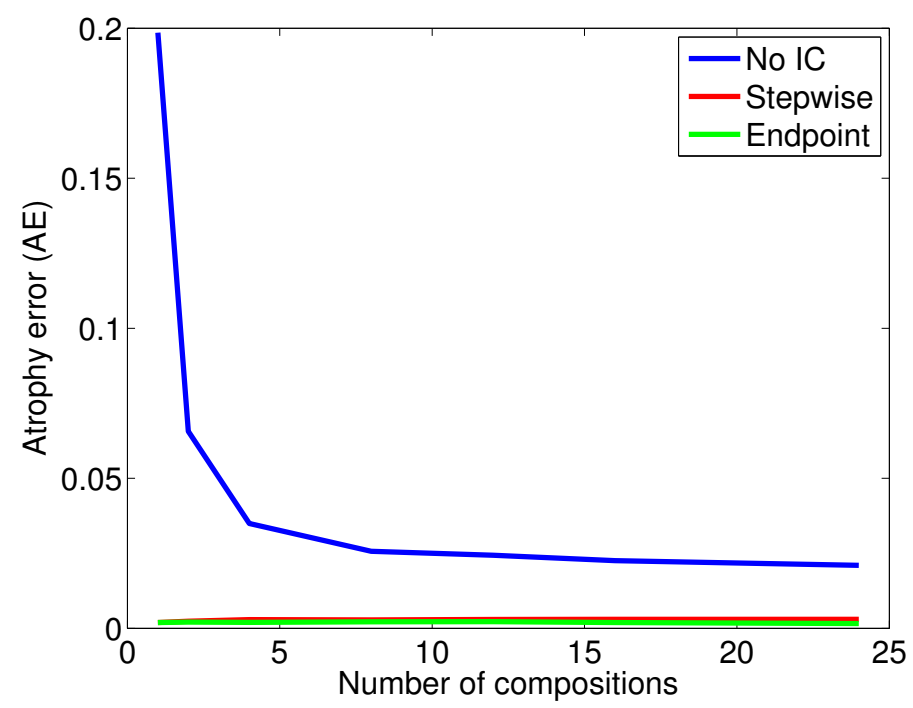

Fig. 3. Mean AE as a function of number of composition. 0.1 is $10 \%$ atrophy error 


\section{Discussion and Conclusions}

In this article, we proposed a stepwise inverse consistent Euler's scheme for diffeomorphic image registration that enforces inverse consistency on the first composition of the transformation as opposed to enforcing it on the endpoint of the full composition, hereby reducing computational complexity. In addition, we presented an implementation of SVF based image registration parametrized by B-splines and in conjunction with the Euler's scheme. The proposed regularization is at a discretization level and can enable symmetric realization of large deformations.

We performed a pairwise comparisons of the stepwise and the endpoint inverse consistency scheme. For comparison we report the registration error (intensity correlation coefficient), displacement offset and bidirectional atrophy difference. The stepwise scheme reduces computational cost while maintaining registration and inverse consistency precision when compared to the endpoint scheme.

This method can be utilized in realizing symmetric large deformations parsimoniously since it parametrizes SVF with the computationally efficient Bsplines. In the future, we would like to investigate the impact of this registration scheme in separating diagnostic groups (Alzheimer's disease and normal controls) based on atrophy in both the whole brain and in subcortical structures.

\section{References}

1. Miller, M.: Computational anatomy: shape, growth, and atrophy comparison via diffeomorphisms. NeuroImage 23(1) (2004) 19-33

2. Tagare, H.D., Groisser, D., Skrinjar, O.: Symmetric non-rigid registration: A geometric theory and some numerical techniques. Journal of Mathematical Imaging and Vision 34(1) (2009) 61-88

3. Beg, M., Miller, M., Trouvé, A., Younes, L.: Computing large deformation metric mappings via geodesic flows of diffeomorphisms. Int. J. Comput. Vis 61 (2005) $139-157$

4. Sommer, S., Lauze, F., Nielsen, M., Pennec, X.: Sparse multi-scale dieomorphic registration: the kernel bundle framework. J. of Mathematical Imaging and Vision 46(3) (2012) 292-308

5. Lorenzi, M., Ayache, N., Frisoni, G., Pennec, X.: 4d registration of serial brain MRI images: a robust measure of changes applied to alzheimer's disease. In: Miccai Workshop on Spatio-Temporal Image Analysis for Longitudinal and Time-Series Image Data, Beijing, China (September 2010)

6. Arsigny, V., Commowick, O., Pennec, X., Ayache, N.: A log-euclidean framework for statistics on diffeomorphisms. In: Medical Image Computing and ComputerAssisted Intervention. Volume 4190. (2006) 924-931

7. Ashburner, J.: A fast diffeomorphic image registration algorithm. NeuroImage 38(1) (2007) 95-113

8. Lorenzi, M., Pennec, X.: Geodesics, parallel transport and one-parameter subgroups for diffeomorphic image registration. International Journal of Computer Vision 105 (2013) 111-127 
9. Bossa, M., Zacur, E., Olmos, S., for the Alzheimer's Disease Neuroimaging Initiative.: Tensor-based morphometry with stationary velocity field diffeomorphic registration: application to adni. NeuroImage 51(3) (2010) 956-969

10. Hernandez, M., Bossa, M.N., Olmos, S.: Registration of anatomical images using paths of diffeomorphisms parameterized with stationary vector field flows. Int. J. Comput. Vis 85 (2009) 291-306

11. Christensen, G.E., Johnson, H.J.: Consistent image registration. IEEE transactions on medical imaging 20 (2001) 568-582

12. Rueckert, D., Aljabar, P., Heckemann, R., Hajnal, J., Hammers, A.: Diffeomorphic registration using b-splines. In: Medical Image Computing and Computer Assisted Intervention (MICCAI). (2006) 702-709

13. Modat, M., Daga, P., Cardoso, M.J., Ourselin, S.: Parametric non-rigid registration using a stationary velocity field. In: IEEE Workshop on Mathematical Methods in Biomedical Image Analysis (MMBIA). (2012) 145 - 150

14. Leow, A., Huang, S., Geng, A., Becker, J., Davis, S., Toga, A., Thompson, P.: Inverse consistent mapping in $3 \mathrm{~d}$ deformable image registration: its construction and statistical properties. In: Information Processing in Medical Imaging. Volume 19. (2005) 493-503

15. Han, X., S, L., Hibbard, V.W.: An efficient inverse-consistent diffeomorphic image registration method for prostate adaptive radiotherapy. In: Prostate Cancer Imaging; Computer-Aided Diagnosis, Prognosis, and Intervention at MICCAI. Volume 6367. (2010) 34-41

16. Avants, B.B., Epstein, C.L., Grossman, M., Gee, J.C.: Symmetric diffeomorphic image registration with cross-correlation: Evaluating automated labeling of elderly and neurodegenerative brain symmetric diffeomorphic image registration with cross-correlation: Evaluating automated labeling of elderly and neurodegenerative brain symmetric diffeomorphic image registration with cross-correlation: Evaluating automated labeling of elderly and neurodegenerative brain. Medical Image Analysis 12(1) (2009) 26-41

17. Darkner, S., Sporring, J.: Generalized partial volume: An inferior density estimator to parzen windows for normalized mutual information. In: Information Processing in Medical Imaging. (2011) 436-447

18. Pai, A., Sørensen, L., Darkner, S., Mysling, P., Jorgensen, D., Dam, E., Lillholm, M., Oh, J., Chen, G., Suhy, J., Sporring, J., Nielsen, M.: Cube propagation for focal brain atrophy estimation. In: IEEE symposium on biomedical imaging. (2013)

19. Rueckert, D., Sonoda, L., Hayes, C., Hill, D., Leach, M.O., Hawkes, D.: Non-rigid registration using free-form deformations: Application to breast MR images. IEEE Transactions on Medical Imaging 18(8) (1999) 712-721 